\title{
Etude des relations entre la dynamique de pré- lèvement d'azote et la dynamique de croissance en matière sèche d'un peuplement de luzerne (Medicago sativa $\mathrm{L}$.)
}

Gilles LEMAIRE, Pablo CRUZ (1), Ghislain GOSSE $\left(^{*}\right)$ \& Michel CHARTIER (*)

I.N.R.A., Station d'Agronomie d'Angers, antenne expérimentale de Lusignan, F 86600 Lusignan.

(*) I.N.R.A., Station de Bioclimatologie, route de St-Cyr, 78000 Versailles.

(') Adresse actuelle : I.N.T.A., AR 2206 Oliveros, République d'Argentine.

Nous avons appliqué à un peuplement de luzerne un modèle d'analyse de la dynamique de prélèvement d'azote en fonction de la croissance en matière sèche préalablement mis au point sur des couverts de graminées fourragères.

Une relation allométrique entre l'azote prélevé et la matière sèche accumulée au cours des différentes repousses peut être proposée. Cette relation est légèrement différente entre la pousse de printemps et les repousses estivales. Cependant, pour chaque numéro de repousse, on obtient une relation identique quels que soient le lieu, l'année et le génotype considérés.

L'accumulation de l'azote dans les feuilles et dans les tiges a été suivie lors de la pousse de printemps et des différentes repousses estivales. On a pu montrer que la consommation d'azote par les feuilles était indépendante du numéro de la repousse tandis que celle des tiges diminuait du printemps à l'été. Pour prendre en compte le comportement différent de ces deux organes, l'évolution du rapport feuilles/tiges en cours de repousse a été exprimée en fonction de la biomasse aérienne totale. Ainsi les différences de prélèvements d'azote entre la pousse de printemps et les repousses d'été se trouvent expliquées par une évolution différente du rapport feuilles/tiges.

Des possibilités de prévision de l'évolution de la qualité fourragère de la luzerne (teneur en protéines, rapport feuilles/tiges) par l'utilisation des relations proposées sont évoquées. Pour que ces prévisions soient opérationnelles, une estimation de la production de matière sèche est nécessaire.

Mots clés additionnels : Teneur en azote, dilution de l'azote, rapport feuilles/tiges, relations d'allométrie.

Relationship between dynamics of nitrogen uptake and dry matter growth for lucerne (Medicago sativa L.).

We have applied a model for the lucerne crop which describes the dynamics of nitrogen uptake during regfowth in relation to dry matter accumulation in the aerial parts. This model was previously established on a grass stand. An allometric relationship between nitrogen uptake and accumulated dry matter during different regrowths is proposed. This relationship was slightly different according to the regrowth : the first regrowth in spring could be distinguished from the three others in summer and autumn. Nevertheless for a given regrowth a very stable relationship was obtained regardless of site, year and genotype.

The accumulation of nitrogen in leaves and in stems was studied during spring and summer regrowth. The relationship between nitrogen uptake and dry matter accumulation for the leaf component appeared independent of the time of year, but for the stem component this relationship was different according to the season : for the spring regrowth the nitrogen consumption by stems appeared higher than for summer regrowths.

In order to take into account the difference in nitrogen content between leaves and stems the change of leaf/stem ratio during each regrowth was examined in relation to dry matter accumulation. During spring regrowth the leaf/stem ratio remained higher than in summer. This explains the higher nitrogen accumulation for a given yield in this season.

Possibilities of prediction of quality of lucerne (protein content, leaf/stem ratio) by using these relationships are discussed.

Additional key words : Nitrogen content, nitrogen dilution, leaf/stem ratio, allometric relationships. 


\section{INTRODUCTION}

La luzerne est la culture qui permet la production de protéines brutes $(\mathrm{N} \times 6,25)$ la plus élevée dans les conditions agronomiques françaises: de 1800 à $2800 \mathrm{~kg} / \mathrm{ha}$. Cependant, la quantité de protéines produite à l'hectare est un critère insuffisant pour l'alimentation animale. Que la luzerne soit consommée directement par les animaux ou bien fasse l'objet d'extractions industrielles de protéines foliaires, la concentration de ces protéines dans la matière sèche récoltée est un paramètre qualitatif déterminant. De nombreuses études ont montré la diminution rapide des teneurs en azote de la luzerne avec l'âge, au cours de la repousse, en relation avec la diminution du rapport feuilles/tiges (WOODMAN \& EVANS, 1935 ; DEMARQUiLly, 1966). Certains auteurs ont proposé des modèles de prévisions de la teneur en protéines de la luzerne basés sur les stades de développement en faisant intervenir les paramètres climatiques tels que la température et le rayonnement (LIU, 1977 ; KALU \& FICK, 1983 ; ONSTAD \& FICK, 1983). Des corrélations négatives entre production de matière sèche et teneurs en azote ont été mises en évidence lors de comparaisons de génotypes, alors qu'entre ces mêmes génotypes il existe des corrélations positives entre production de protéines et production de matière sèche (GUY et al., 1969).

Toutes ces observations laissent supposer l'existence d'une relation plus ou moins étroite entre l'accumulation d'azote dans les parties aériennes et leur croissance en matière sèche. Une telle relation a déjà été mise en évidence pour des peuplements de graminées fourragères : on a pu formuler une loi décrivant la dilution de l'azote prélevé dans la matière sèche élaborée au cours de la croissance (SALETTE \& LEMAIRE, 1981). Cette loi de type :

$$
\text { N p. } 100=\alpha(\mathrm{MS})^{-\beta}(1)
$$

où $\mathrm{N}$ p. 100 est la teneur en azote des parties aériennes à un moment donné de la repousse et MS la quantité de matière sèche en $t /$ ha des parties aériennes au même instant, peut se traduire par la relation mathématiquement équivalente :

$$
\mathrm{N} \exp =10 \alpha(\mathrm{MS})^{1-\beta}(2)
$$

reliant $\mathrm{N}$ exp, la quantité d'azote contenue dans les parties aériennes à un moment donné de la repousse, à la production de MS (le coefficient 10 étant nécessaire pour exprimer $\mathrm{N}$ exp en $\mathrm{kg} / \mathrm{ha}$ ).

L'expression (2) représente, en fait, une relation d'allométrie entre les prélèvements d'azote et la croissance en MS. Le coefficient 1- $\beta$ est le rapport des vitesses relatives de prélèvement d'azote et des vitesses relatives de croissance; le coefficient $10 \alpha$ représente la quantité d'azote prélevée pour l'élaboration de la $1^{\text {re }} t$ de MS lors de la repousse. L'utilisation d'un tel modèle sur les peuplements de graminées fourragères a permis de rendre parfaitement compte, en conditions de nutrition azotée non limitantes, à la fois des effets du milieu (différences entre années) et des différences entre génotypes (LEMAIRE \& SALETTE, 1984a et b).

L'objectif de ce travail a été de vérifier si ce modèle, mis au point sur des peuplements de grami- nées fourragères, pouvait s'appliquer à des peuplements de luzerne et d'analyser en quoi la composition morphologique du couvert végétal (rapport feuillestiges) pourrait en influencer les paramètres.

\section{MATÉRIEL ET MÉTHODES}

La croissance de la luzerne a été étudiée en 2 lieux, La Minière (Yvelines) et Lusignan (Vienne), sur plusieurs années. Les parcelles ont été semées au printemps, mais les études ne concernent que les 2 années après l'année d'installation. En règle générale, le calendrier d'exploitation de la luzerne est le suivant :

pousse de printemps... coupe le 10-20 mai

$1^{\text {re }}$ repousse

$2^{\mathrm{e}}$ repousse

$3^{\mathrm{e}}$ repousse

$$
\begin{gathered}
-\quad 25 \text { juin-5 juillet } \\
-\quad 10-15 \text { août } \\
-\quad 30 \text { septembre - } \\
5 \text { octobre. }
\end{gathered}
$$

La croissance en MS de chaque pousse ou repousse est suivie par des prélèvements hebdomadaires de $3 \mathrm{~m}$ linéaires sur chacun des 5 blocs que comportent les essais. La hauteur de coupe est de $6 \mathrm{~cm}$. Sur chaque prélèvement, on détermine le poids de matière verte et on constitue un échantillon pour détermination des teneurs en MS, puis broyage et analyse. La teneur en azote est déterminée pour chacun des 5 prélèvements. Sur les 2 blocs donnant les productions de matière verte les plus proches de la moyenne, on fait un prélèvement de $0,50 \mathrm{~m}$ linéaire supplémentaire sur une ligne adjacente afin d'effectuer une séparation entre feuilles (foliole + pétiole) et tiges. Les feuilles et les tiges ainsi obtenues sont séchées et broyées séparément, puis analysées.

Ainsi, chaque semaine, au cours des différentes repousses, nous avons pu déterminer les variables suivantes :

\begin{tabular}{lccc} 
& Feuilles & Tiges & $\begin{array}{l}\text { Plante } \\
\text { entière }\end{array}$ \\
\hline $\begin{array}{l}\text { Matière sèche en t.ha } \text { ha }^{-1} \\
\begin{array}{l}\text { Teneur en azote en } \% \text { de } \\
\text { MS }\end{array}\end{array}$ & MSf & MSt & MST \\
$\begin{array}{l}\text { Prèlèvements d'azote en } \\
\text { kg.ha }{ }^{-1}\end{array}$ & Nf $\%$ & NT $\%$ \\
\hline \hline
\end{tabular}

Les mesures ont été effectuées simultanément en 1980 et 1981 à Lusignan et à La Minière sur la variété " du Puits », puis sur un autre essai, en 1982 et 1983, à Lusignan sur les seules repousses d'été de la variété « Europe » en parcelles irriguées. Enfin, nous avons pu également utiliser des données, au niveau global, des parties aériennes sur divers essais non prévus initialement à cet effet.

A Lusignan, le sol est un sol brun lessivé sur limon, superposé à une argile rouge à pisolithes. Le $\mathrm{pH}$ est de 6,5 environ. Ce sol ne présente aucun facteur pédologique limitant le développement de la luzerne. Seul un déficit hydrique marqué, associé à des fortes ETP, peut, certaines années, limiter fortement la croissance estivale.

A La Minière, le sol est un limon des plateaux profond sur argile à meulière $(1,20 \mathrm{~m})$. Le pH est de 
6,8 environ. Ce sol possède une réserve utile de l'ordre de $18 \mathrm{~mm}$ par tranche de $10 \mathrm{~cm}$ de sol. Le déficit hydrique estival ne limite qu'exceptionnellement la croissance de la luzerne.

Dans ces 2 situations, les excès d'eau de début de printemps sont susceptibles de limiter le développement de rhizobiums de la luzerne à cette période.

\section{RÉSULTATS ET DISCUSSIONS}

\section{A. Analyse au niveau des parties aériennes globales}

1. Comparaison entre lieux et entre années, selon le numéro de repousse

Pour chaque repousse étudiée, nous disposons d'une série de valeurs de MST, NT p. 100 et NT exp nous permettant d'ajuster :

la relation (1) NT p. $100=\alpha(\text { MST })^{-\beta}$ ou la relation (2) NT exp $=10 \alpha(\mathrm{MST})^{1-\beta}$ en utilisant les régressions orthogonales sur les logarithmes des variables initiales.

Les relations (1) et (2) étant mathématiquement équivalentes, nous avons choisi pour la présentation des résultats de privilégier la relation (2), plus parlante sur le plan agronomique, puisque décrivant la relation d'allométrie entre la croissance en MS et les prélève- ments d'azote des parties aériennes, l'évolution des teneurs en azote décrites par la relation (1) devant plutôt être considérée comme en étant la conséquence.

La comparaison des différentes relations obtenues pose un problème statistique non résolu de manière satisfaisante sur le plan théorique. DAGNELIE (1969) a indiqué une méthode de détermination de l'intervalle de confiance du coefficient de régression orthogonale. Nous avons utilisé cette méthode pour comparer les différentes valeurs du coefficient d'allométrie (1- $\beta$ ). En cas de parallélisme des régressions, il convient alors de comparer les valeurs du coefficient $10 \alpha$. Il n'existe pas de méthode rigoureuse pour effectuer ces comparaisons (DAGNELIE, 1969). Cependant, les points ayant servi à calculer les différentes régressions sont en fait des moyennes de 5 prélèvements sur lesquels étaient déterminées simultanément la production de MS et la teneur en azote. Nous avons pu estimer à travers toutes nos données l'intervalle de confiance de la moyenne de la teneur en azote des 5 répétitions. Pour la luzerne, les valeurs se trouvaient comprises en règle générale entre 0,3 et $0,4 \mathrm{p} .100$ d'azote. Cette précision correspond à une variation de 3 à $4 \mathrm{~kg}$ d'azote/ha pour le coefficient $10 \alpha$.

La figure 1 représente les relations allométriques obtenues pour la pousse de printemps et les 3 repousses suivantes de la variété « du Puits" à Lusignan et à La Minière, en 1980 et 1981. Il apparaît clairement que les différences entre lieux et entre années sont très
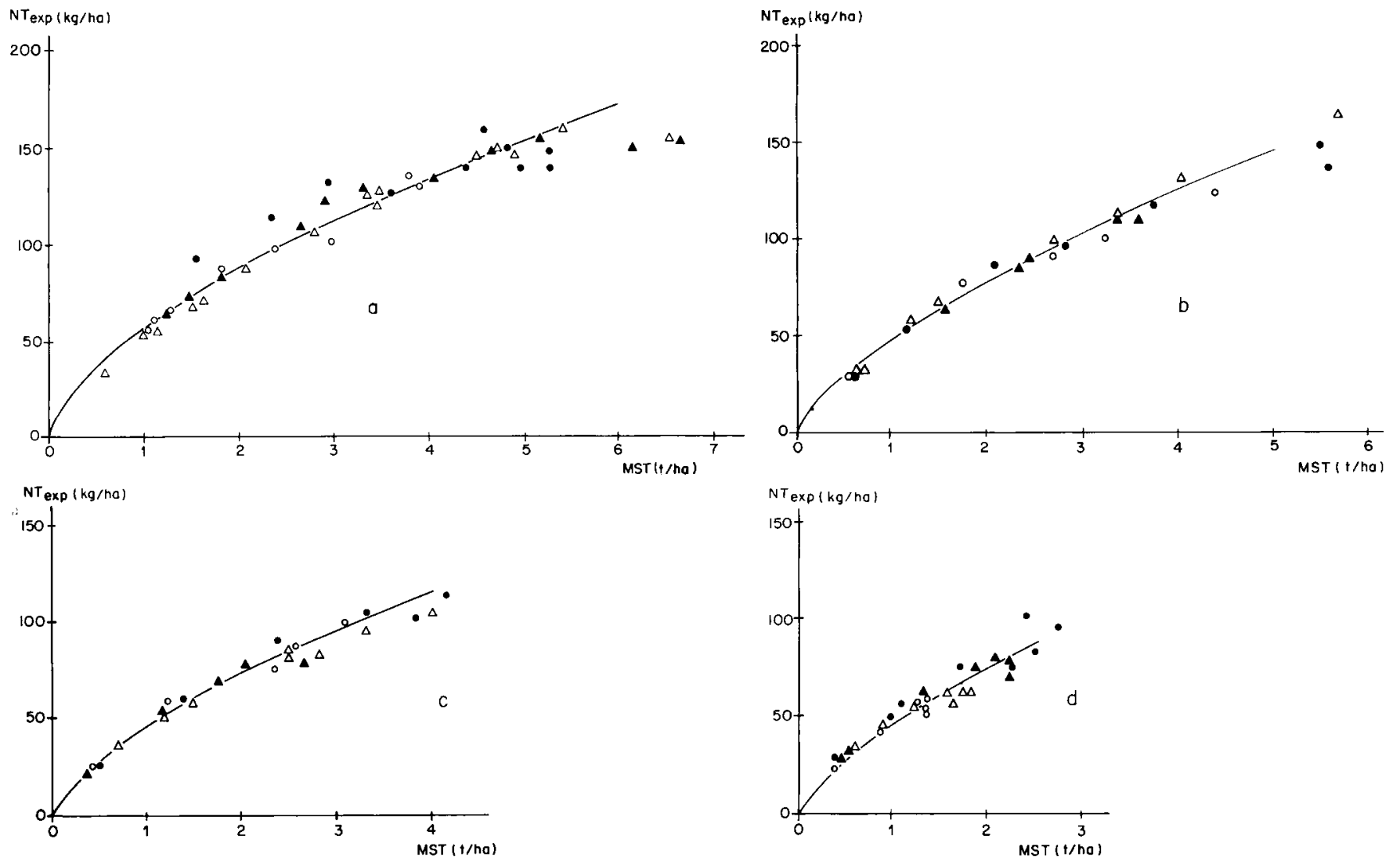

Figure 1

growth of lucerne for different regrowths at Lusignan and La Minière.

Relation allométrique entre prélèvements d'azote et croissance en matière sèche de la luzerne pour différentes repousses à Lusignan et à La Minière.

Allometric relationship between nitrogen uptake and dry matter

(a) pousse de printemps; spring growth; La Minière, Lusignan.

(b) Ire repousse; Ist regrowth; $\quad\} \triangle 1980 ; \quad \Delta 1980$.

(d) $3^{e}$ repousse; 3rd regrowth; $1981 ; 01981$ 
faibles et une régression commune a pu être calculée pour chaque repousse.

Cependant, on peut noter sur la figure la une différenciation assez sensible pour l'année 1981 à Lusignan qui se caractérise par une accumulation de $10 \mathrm{~kg}$ d'azote supplémentaires en début de croissance et un retour à la normale en fin de croissance. Ce comportement peut être rapproché d'observations effectuées cette même année sur des peuplements de graminées où nous avions mis en évidence une minéralisation très exceptionnelle de l'azote du sol en début de printemps.

On peut remarquer egalement en fin de croissance, au-delà d'une production de $5 \mathrm{t}$ de $\mathrm{MS}$, un net fléchissement des prélèvements d'azote. Ceci correspond à des phénomènes de sénescence accentués par des débuts de verse.

Le tableau 1 permet de comparer les coefficient $\alpha$ et $\beta$ obtenus pour les différentes repousses. La pousse de printemps montre une consommation d'azote plus importante que les repousses suivantes, à un même niveau de croissance. Ceci est déterminé par la valeur du coefficient $10 \alpha$ qui mesure en fait la consommation d'azote correspondant à l'édification de la $1^{\text {re }} \mathbf{t}$ de MS : $55 \mathrm{~kg} / \mathrm{ha}$ au printemps contre 46 à $48 \mathrm{~kg} / \mathrm{ha}$ aux autres repousses. Cette variation est de 2 à 3 fois supérieure à celle qui serait explicable par la variabilité de la détermination des teneurs en azote; nous admettrons donc qu'elle est bien caractéristique du numéro de la repousse.

La valeur du coefficient $(1-\beta)$ a tendance à être légèrement plus faible pour la pousse de printemps notamment lorsqu'on la compare à celle obtenue pour la $1^{\text {re }}$ repousse. L'atténuation des prélèvements d'azote semble donc plus rapide au printemps, ce qui correspond, comme nous l'avons indiqué dans l'examen de la figure 1a, à l'importance des phénomènes de sénescence en fin de croissance.

\section{Comparaison entre génotypes de luzerne}

Nous avons pu comparer ces relations obtenues sur la variété "du Puits» avec celles obtenues sur d'autres variétés dans des essais différents à I.usignan. Nous avions à notre disposition :

- des mesures de croissance sur repousses estivales en irrigué sur les variétés «Lutèce » et «Europe » en 1982 et 1983 . Le tableau 2 permet de comparer les

TABLEAU

Comparaison des coefficients $10 \alpha$ et $1-\beta$ de la relation $N T \exp =10 \alpha(M S T)^{1-\beta}$ pour les différentes repousses. Comparison of coefficients $10 \alpha$ and $1-\beta$ for different regrowths.

$$
\begin{array}{lll}
10 \alpha & 1-\beta & \mathrm{R}^{2} \quad \begin{array}{l}
\text { Intervalle de confiance } \\
\text { de }(1-\beta) ; \mathrm{P}=0,05
\end{array}
\end{array}
$$

\begin{tabular}{lllll}
\hline Pousse de printemps & 55 & 0,64 & 0,978 & $0,61-0,67$ \\
\hline $1^{\text {re } \text { repousse }}$ & 48 & 0,69 & 0,996 & $0,68-0,71$ \\
\hline $2^{\text {e } \text { repousse }}$ & 46 & 0,67 & 0,970 & $0,62-0,72$ \\
\hline $3^{\text {e repousse }}$ & 47 & 0,71 & 0,968 & $0,66-0,76$ \\
\hline
\end{tabular}

TABLEAU 2

Comparaison des coefficients $10 \alpha$ et $I-\beta$ de la relation $N T$ exp $=10 \alpha(M S T)^{I-\beta}$ obtenues avec les variétés "Lutèce " et “Europe 》 (1982 et 1983) avec la variété « du Puits» (1980-1981) pour l'ensemble des premières et deuxièmes repousses confondues.

Comparison of coefficients $10 \alpha$ and I- $\beta$ obtained with 'Lutèce' and 'Europe' (1982-1983) and with 'du Puits' (1980-1981). Means of the first and second regrowths.

\begin{tabular}{lcccc}
\cline { 2 - 5 } & $10 \alpha$ & $1-\beta$ & $\mathrm{R}^{2}$ & $\begin{array}{c}\text { Intervalle de confiance } \\
\text { de }(1-\beta)\end{array}$ \\
\hline Lutèce & 47 & 0,70 & 0,973 & $0,66-0,75$ \\
\hline Europe & 50 & 0,66 & 0,954 & $0,61-0,72$ \\
\hline du Puits & 46 & 0,69 & 0,971 & $0,64-0,75$ \\
\hline
\end{tabular}

ajustements obtenus avec ces 2 variétés avec ceux de la variété «du Puits» pour l'ensemble des $1^{\text {res }}$ et $2^{\text {es }}$ repousses. Les différences entre les valeurs de 1- $\beta$ ne sont pas significatives. L'écart entre les valeurs de $10 \propto$ d'«Europe » et du Puits n'excède pas $4 \mathrm{~kg}$ d'azote/ha ;

- des mesures de croissance comparées entre 3 autres variétés «Europe », « Maris Kabul » et «Magali » pour la pousse de printemps 1982. La figure 2 permet de confirmer la stabilité du modèle pour une gamme de génotypes plus large. Seule "Magali » semble se distinguer légèrement par un ralentissement plus marqué des prélèvements d'azote en fin de repousse, ce qui se traduit par une valeur plus faible du coefficient (1- $\beta$ ) (tabl. 3 ).

\section{Comparaison avec les peuplements de graminées}

La grande stabilité des relations, pour un même numéro de repousse, entre années, entre lieux et entre

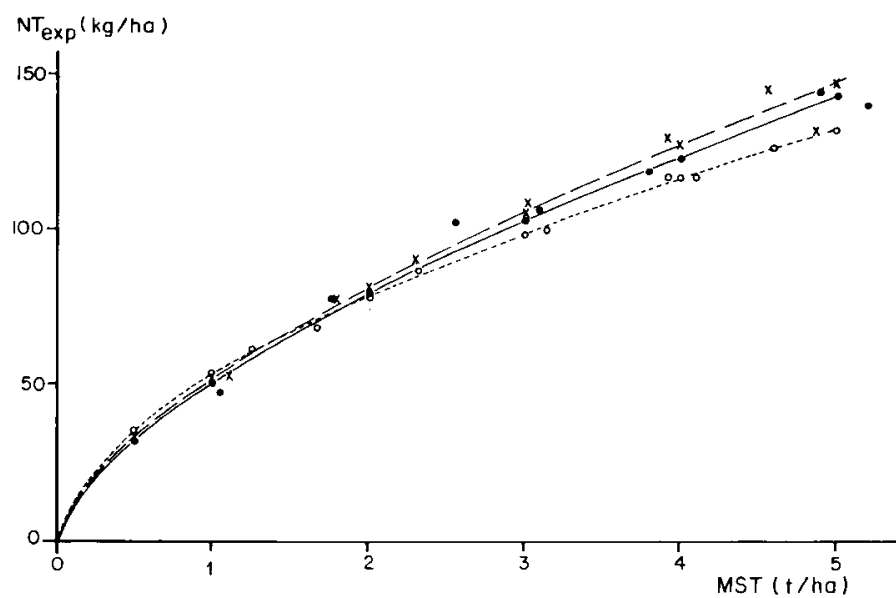

Figure 2

Relation allométrique entre prélèvement d'azote et croissance en matière sèche lors de la pousse de printemps pour 3 variétés de luzerne.

Allometric relationship between nitrogen uptake and dry matter growth for spring growth and three varieties.

$\begin{array}{lll}\text { Europe } & \text { NT exp }=51(M S T)^{0,65} & R^{2}=0,974 \\ \text { Magali } & \text { NT exp }=53(M S)^{0,57} & R^{2}=0,996 \\ \times \text { Maris Kabul } & \text { NT exp }=52(M S T)^{0,65} & R^{2}=0,978\end{array}$




\section{TABLEAU 3}

Comparaison des coefficients $10 \alpha$ et $1-\beta$ de la relation $N T \exp =10 \alpha(M S T)^{I-\beta}$ obtenus avec les variétés «Europe », «Magali » et « Maris-Kabul » lors de la pousse de printemps 1982. Comparison of coefficients $10 \alpha$ and 1- $\beta$ obtained with 'Europe', 'Magali' and 'Maris-Kabul' for the spring regrowth in 1982.

$\begin{array}{llll}10 \alpha & 1-\beta & \mathbf{R}^{2} \quad \text { Intervalle de confiance }\end{array}$ de $(1-\beta)$

\begin{tabular}{lcccc}
\hline Europe & 51 & 0,65 & 0,974 & $0,60-0,70$ \\
\hline Magali & 53 & 0,57 & 0,996 & $0,56-0,59$ \\
\hline Maris-Kabul & 52 & 0,65 & 0,978 & $0,60-0,70$ \\
\hline
\end{tabular}

génotypes différents, nous a incités à les comparer à celles obtenues sur les peuplements de graminées pérennes avec un niveau de fertilisation azotée non limitant et publiées antérieurement (LEMAIRE \& SAletTe, $1984 a$ et $b$ ). Pour 6 années d'expérimentation et pour la pousse de printemps, nous obtenions pour la fétuque élevée cv. "Ludelle » la relation suivante :

$\mathrm{N} \exp =48(M S)^{0,68}\left(\mathrm{R}^{2}=0,962\right)$, ce qui apparaît assez proche des ajustements obtenus sur luzerne.

Une possibilité de comparaison plus rigoureuse a été possible en utilisant les données de la croissance de la luzerne (cv. Lutèce) et du dactyle (cv. "Lutetia ») mesurée en 1982 à Lusignan sur les parcelles « culture pure », dans un même essai qui avait pour but l'étude du comportement en association de ces 2 espèces. La figure 3 nous montre la différence de comportement entre luzerne et dactyle qui ne se perçoit réellement qu'en fin de repousse, notamment lors de la pousse de printemps. Ainsi, malgré des voies d'approvisionnement en azote très différentes, les prélèvements d'azote par les parties aériennes d'une luzerne semblent déterminés par leur propre dynamique de croissance selon une relation similaire à celle obtenue sur des graminées bien fertilisées.

\section{B. Analyse de l'effet de la composition morphologique}

La stabilité des relations observées nous a incités à examiner quel pouvait être le poids de la composition morphologique du couvert végétal, étant donné les différences de teneur en azote entre les feuilles et les tiges.

La séparation en feuilles et en tiges de la matière sèche récoltée sur certains essais nous a permis d'appliquer le modèle de prélèvement d'azote au niveau de ces 2 compartiments.

La figure 4 montre qu'au niveau des feuilles, il n'est pas possible de mettre en évidence des différences entre numéro de repousse à Lusignan et que les données de 1980 à La Minière ne s'en distinguent pas significativement. Le coefficient d'allométrie (1- $\beta)$ relativement élevé $(0,87$ environ) montre que la teneur des feuilles diminue peu au cours des repousses, de $5,6 \%$ en moyenne en tout début de repousse à 4,6\% environ en fin de repousse. Ceci confirme les données de WOODWARD \& SHEEHY (1979) qui avaient en outre montré que la teneur en azote des jeunes feuilles (se situant dans les 15 premiers $\mathrm{cm}$ du couvert) était relativement constante tout au long de la repousse (de 5,8 à $5,5 \%$ ), alors que celle des feuilles de la base, plus âgées, diminuait jusqu'à $3,5 \%$ en fin de cycle.

La figure 5 représente les relations obtenues pour les prélèvements d'azote par les tiges en 1980 et 1981 à Lusignan. On peut regrouper dans un même comportement la pousse de printemps et la $1^{\text {re }}$ repousse d'été,

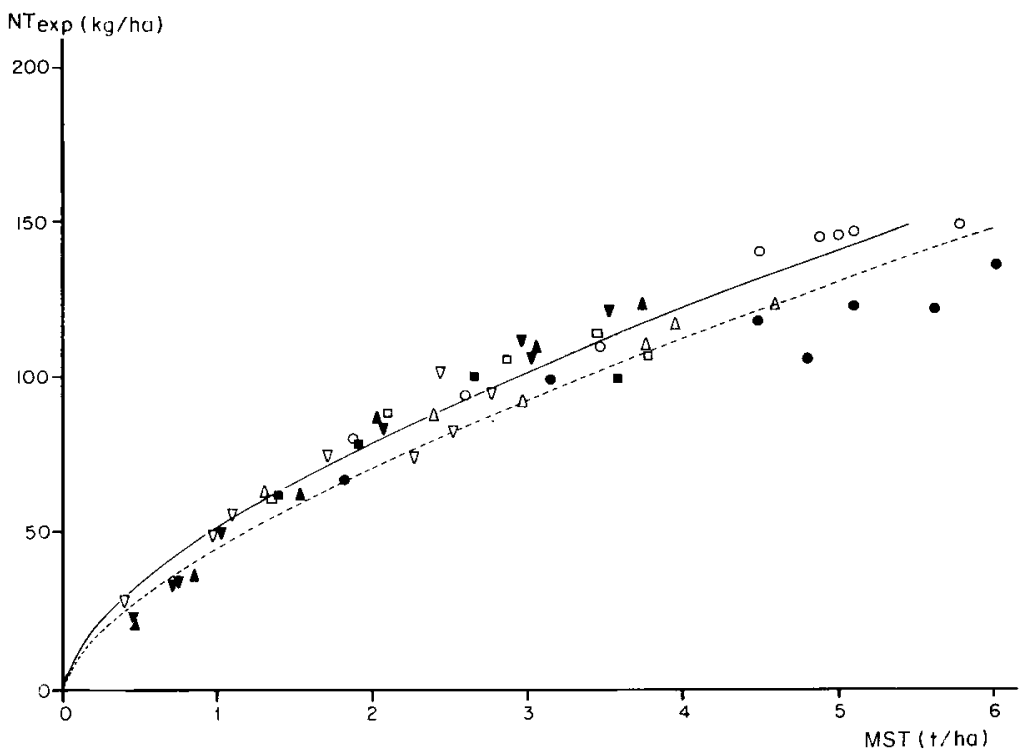

Figure 3

Comparaison des relations allométriques entre prélèvements d'azote et croissance en matière sèche obtenus sur des peuplements de luzerne et de dactyle pour l'ensemble des repousses.

Comparison of allometric relationship obtained on lucerne and cocksfoot for different regrowths.
Luzerne: $\left(\longrightarrow\right.$ ) pousse de printemps; $\square I^{\text {re }}$ repousse; $\triangle 2^{e}$ repousse $; \nabla 3^{2}$ repousse.

$N T \exp =54(M S)^{0,59} \quad R^{2}=0,976$.

Dactyle : (---) pousse de printemps; Ire repousse ; $^{\text {re }}$

$\Delta 2^{e}$ repousse; $\nabla 3^{e}$ repousse.

$N T \exp =44(M S)^{0,68} \quad R^{2}=0,936$. 


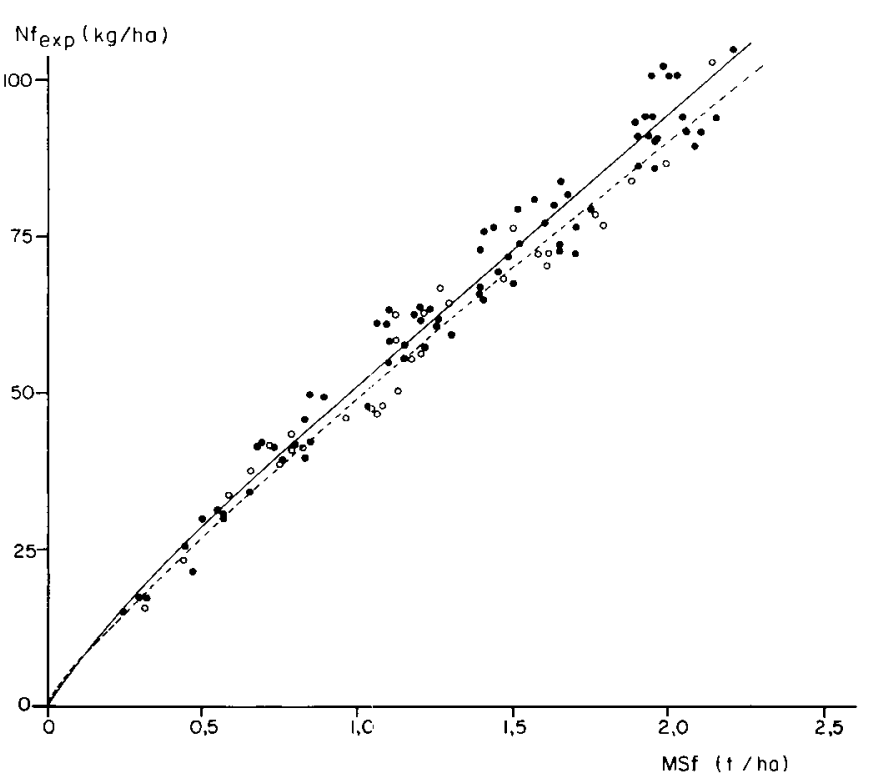

Figure 4

Relation entre les prélèvements d'azote et la croissance en matière sèche des feuilles de luzerne pour l'ensemble des repousses à Lusignan et à La Minière.

Relationships between nitrogen uptake and dry matter growth of leaves of lucerne for different regrowths at Lusignan and La Minière.

Ensemble des repousses Lusignan 1980-81 ( $\longrightarrow$ ) : Nf exp $=52(M S f)^{0,87} \quad R^{2}=0,963$.

Ensemble des repousses La Minière 1980 ( $\bigcirc---)$ :

$N f \exp =50(M S f)^{0,86} \quad R^{2}=0,967$.

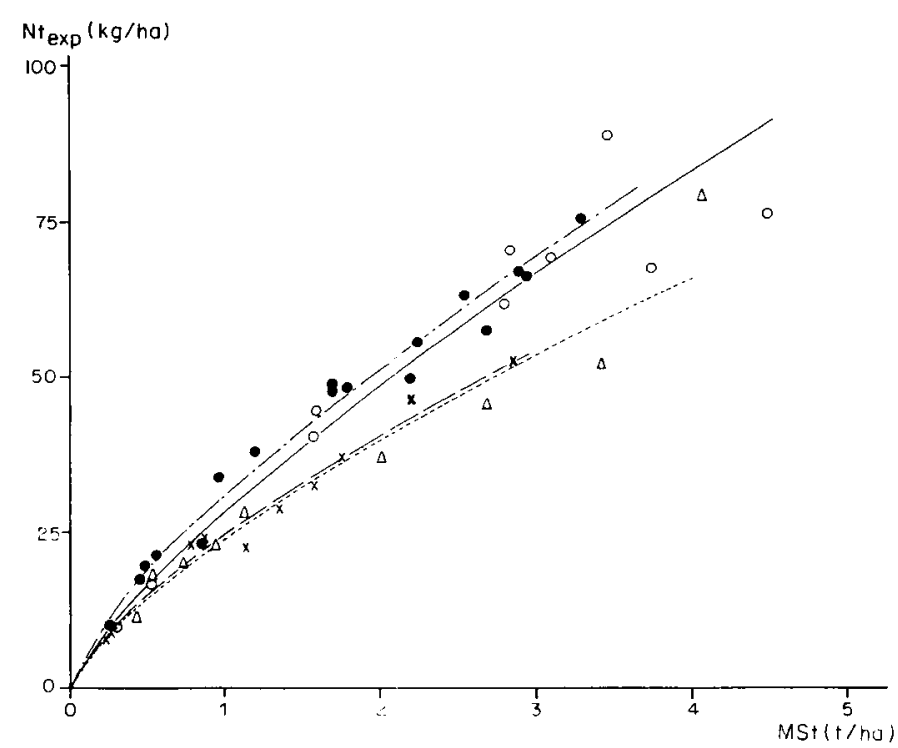

Figure 5

Relations entre les prélèvements d'azote et la croissance en matière sèche des tiges de luzerne pour les différentes repousses à Lusignan en 1980 et 1981.

Relationships between nitrogen uptake and dry matter growth of stems of lucerne for different regrowths at Lusignan in 1980 and 1981.

\begin{tabular}{|c|c|c|}
\hline pousse de printemps & $N t \exp =31(M S t)^{0,74}$ & $R^{2}=0,982$ \\
\hline Ire repousse & $N t \exp =28(M S t)^{0,78}$ & $R^{2}=0,974$ \\
\hline $\begin{array}{l}\triangle \\
2^{e} \text { repousse }\end{array}$ & $N t \exp =24(M S t)^{0,73}$ & $R^{2}=0,961$ \\
\hline $3^{e}$ repousse & $N t_{\exp }=24(\mathrm{MSt})^{0,71}$ & $R^{2}=0,978$ \\
\hline
\end{tabular}

d'une part, et les $2^{\mathrm{e}}$ et $3^{\mathrm{e}}$ repousses, d'autre part. La consommation d'azote par les tiges est, bien entendu, plus faible que celle des feuilles : de 24 à $31 \mathrm{~kg}$ d'azote pour la $1^{\text {re }} \mathrm{t}$ de $\mathrm{MS}$ élaborée pour les tiges contre $50 \mathrm{~kg}$ environ pour les feuilles. D'autre part, le coefficient d'allométrie (1- $\beta$ ), plus faible au niveau des tiges (de 0,71 à 0,74 selon les repousses), indique une diminution plus prononcée des teneurs en azote des tiges au cours de la repousse (tabl. 4). Les données de La Minière, en 1980, confirment globalement ces observations avec, cependant, une plus grande différence entre les repousses ; notamment la $3^{\mathrm{e}}$ repousse présente un coefficient $1-\beta$ très bas, ce qui est le signe d'une diminution très rapide des prélèvements d'azote.

pousse de printemps $=\mathrm{Nt} \exp =\mathbf{3 0}(\mathrm{MSt})^{0,76} \mathrm{R}^{2}=0,996$ $1^{\text {re }}$ repousse $\quad=N t \exp =27(\mathrm{MSt})^{0,67} \mathrm{R}^{2}=0,996$ $2^{\mathrm{e}}$ repousse $\quad=\mathrm{Nt} \exp =21(\mathrm{MSt})^{0,64} \mathrm{R}^{2}=0,984$ $3^{\mathrm{e}}$ repousse $\quad=\mathrm{Nt} \exp =17(\mathrm{MSt})^{0,46} \mathrm{R}^{2}=0,947$

L'examen de l'évolution des rapports feuilles/tiges au cours des différentes repousses devrait nous permettre de reconstituer les relations obtenues au niveau de la matière sèche aérienne totale à partir des relations élémentaires au niveau des feuilles et des tiges et expliquer ainsi les différences constatées entre les pousses de printemps et les repousses suivantes.

Nous avons cherché à relier l'évolution du rapport feuilles/tiges à la croissance en MS pour les différentes repousses. Les équations obtenues traduisent en fait une dilution de la masse de feuille dans une masse de tige croissante. La figure 6 montre qu'il y a une cinétique différente de l'évolution du rapport feuilles/tiges au printemps par rapport aux repousses d'été : la valeur de ce rapport en début de croissance est 2 fois plus élevée au printemps, 3,5 contre 1,7, pour une production de $1 \mathrm{t}$ de MST/ha, alors qu'elle devient identique $(0,6)$ pour une production de $6 \mathrm{t}$ de $\mathrm{MST} / \mathrm{ha}$.

Pour la pousse de printemps, nous avons proposé une équation unique bien que la figure 6a suggère des rapports feuilles/tiges légèrement plus élevés à Lusignan qu'à La Minière. Mais, dans l'état actuel, il nous est impossible de déterminer si cet écart est relié effectivement à un effet du milieu ou bien s'il s'agit d'une légère différence de manipulation dans la sépa-

\section{TABLEAU 4}

Comparaison des coefficients $10 \alpha$ et $1-\beta$ de la relation $N t \exp =10 \alpha(M S t)^{l-\beta}$ pour les différentes repousses à Lusignan en 1980 et 1981.

Comparison of coefficients $10 \alpha$ and $1-\beta$ for stem component at Lusignan in 1980-1981.

$10 \alpha \quad 1-\beta \quad R^{2} \quad$ Intervalle de confiance de $(1-\beta)$

\begin{tabular}{lllll}
\hline Pousse de printemps & 31 & 0,74 & 0,982 & $0,69-0,79$ \\
\hline 1 $^{\text {re }}$ repousse & 28 & 0,78 & 0,974 & $0,69-0,88$ \\
\hline 2 $^{\mathrm{e}}$ repousse & 24 & 0,73 & 0,961 & $0,62-0,87$ \\
\hline 3e repousse $^{\mathrm{e}}$ & 24 & 0,71 & 0,978 & $0,63-0,80$ \\
\hline \hline
\end{tabular}



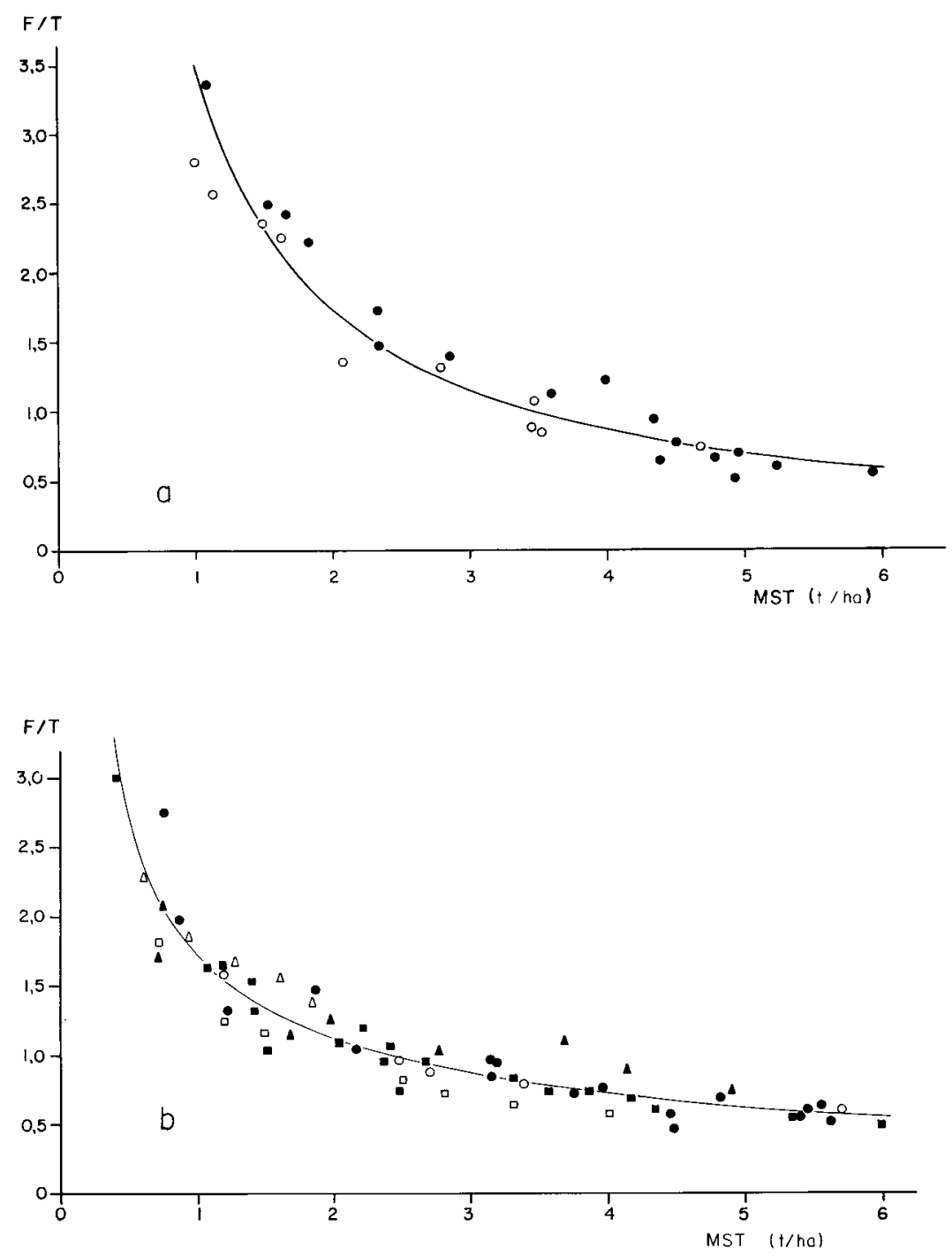

Figure 6

Relation entre le rapport feuilles/tiges et la croissance en matière sèche au cours des différentes repousses à Lusignan 1980-1981 et à La Minière 1980.

Relationships between leaf/stem ratio and dry matter growth for different regrowths at Lusignan and La Minière.

ration des feuilles et des tiges entre les 2 sites expérimentaux.

Pour les autres repousses, cette différence n'apparaît plus, et la figure $6 \mathrm{~b}$ représente l'évolution du rapport feuilles/tiges pour toutes les repousses estivales des 2 sites confondus.

L'interprétation de ces observations pourrait être la suivante :

Le rapport feuilles/tiges initialement plus élevé de la pousse de printemps s'explique par le fait que, l'élongation des tiges étant fortement sous la dépendance de la photopériode (GOSSE et al., 1982), la luzerne commence à émettre une quantité importante de feuilles en " rosette " au début du printemps avant que la croissance des tiges ne s'accélère. Les 2 repousses suivantes étant à peu près symétriques par rapport au solstice d'été ne présentent pas de différence sensi. ble d'évolution du rapport feuilles/tiges; la tendance de la $3^{\mathrm{e}}$ repousse à avoir un rapport feuilles/tiges légè-

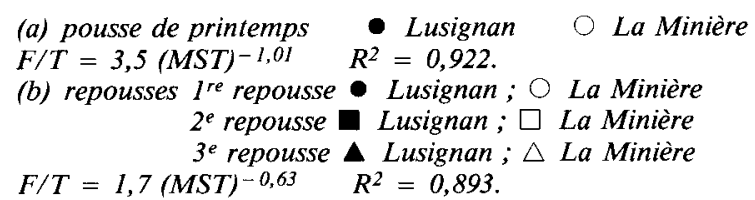

rement plus élevé en fin de croissance marque le retour à des photopériodes plus courtes (fin aoûtseptembre).

Ainsi semble s'expliquer assez clairement la dynamique de prélèvement d'azote plus faible de la $1^{\text {re }}$ repousse comparativement à la pousse de printemps.

Par contre, les différences observées entre la $1^{\text {re }}$ et la $2^{\mathrm{e}}$ repousse (fig. 3 ) ne peuvent s'expliquer que par les différences de consommation d'azote par les tiges constatées sur la figure 5, puisque l'évolution du rapport feuilles/tiges est similaire dans les 2 cas. Ces consommations plus faibles d'azote par les tiges lors de la $2^{\mathrm{e}}$ repousse peuvent s'interpréter :

- soit par une lignification plus rapide des tiges due à l'effet des températures plus élevées de juilletaoût (DEINUM, 1984) ;

- soit par une moindre fourniture d'azote à cette période. La plante optimiserait alors sa consomma- 
tion d'azote au niveau des feuilles (ce qui est attesté par l'absence de différence constatée sur la figure 4) et laisserait supporter aux tiges les effets d'une offre globale en azote plus réduite.

Ces 2 hypothèses ne sont pas contradictoires et peuvent contribuer toutes les 2 à l'explication des différences constatées.

\section{CONCLUSION}

Il apparaît qu'une modélisation de la teneur en protéines et de la production de protéines $(\mathrm{N} \times 6,25)$ d'une culture de luzerne soit possible à partir de la seule connaissance de la quantité de matière sèche récoltée pour un numéro de repousse donné. Le modèle semble stable quels que soient l'année, le lieu et la variété, du moins dans les gammes étudiées.

Une sécheresse modérée ne semble pas remettre en cause les relations proposées même si elle ralentit la croissance en matière sèche ; cependant, si elle s'accentue, elle peut perturber la relation initiale en limitant la nutrition azotée.

L'aspect prédictif de ce modèle pourrait avantageusement être valorisé en le couplant au modèle prévisionnel de la croissance en matière sèche proposé par GOSSE et al. (1984).

Enfin, l'utilisation de telles relations obtenues en conditions de nutrition azotée apparemment non limitantes et en absence de déficit hydrique devrait servir de base de référence dans le diagnostic de situations dans lesquelles on suppose que le niveau d'alimentation azotée ait pu être limitant pour des raisons diverses.

De plus, des études actuellement en cours sur l'effet d'un stress hydrique, tant sur le modèle de croissance en matière sèche que sur le modèle de prélèvement d'azote, devraient permettre d'intégrer le facteur sécheresse dans les modèles de prévision de production et de qualité de la luzerne.

Reçu le 20 novembre 1984. Accepté le 27 mars 1985.

\section{RÉFÉRENCES BIBLIOGRAPHIQUES}

Dagnelie P., 1969. Théorie et méthodes statistiques, tome 2. E. J. Duclot, S. A. Gembloux, 463 p.

Deinum B., 1984. Chemical composition and nutritive value of herbage in relation to climate, 338-350 ; in Riley H. \& Skjelvag O. : Proceedings of 10th general meeting of European Grassland Federation. As, Norway. 26-30 juin 1984.

Demarquilly C., 1966. Valeur alimentaire de la luzerne. Fourrages, 26, 12-33.

Gosse G., Chartier M., Lemaire G., Guy P., 1982. Facteurs climatiques et production chez la luzerne. Fourrages, 90, 113-135.

Gosse G., Chartier M., Lemaire G., 1984. Mise au point d'un modèle de prévision de production pour une culture de luzerne. $C$. R. Acad. Sci. Paris, 298, Série III, 18, 541-544.

Guy P., Genier G., Porcheron A. M., 1969. Relation between quality and yield in lucerne. Rapport of the meeting of Eucarpia Fodder Crops, section WPBS, Aberystwyth, April 15th-17th, 51-55.

Kalu B. A., Fick G. W., 1983. Morphological stage of development as a predictor of alfalfa herbage quality. Crop Sci., 23, 1167-1172.

Lemaire G., Salette J., 1984a. Relation entre dynamique de croissance et dynamique de prélèvement d'azote pour un peuplement de graminées fourragères. I. Etude de l'effet du milieu. Agronomie, 4 (5), 423-430.

Lemaire G., Salette J., 1984b. Relation entre dynamique de crois sance et dynamique de prélèvement d'azote pour un peuplement de graminées fourragères. II. Etude de la variabilité entre génotypes. Agronomie, 4 (5), 431-436.

Liu B. W. Y., 1977. Statistical models for production of alfalfa quality. Ph. D. Thesis, Cornell Univ.

Onstad D. W., Fick G. W., 1983. Predicting crude protein in vitro, true digestibility and leaf proportion in alfalfa herbage. Crop Sci., 23, 961-964.

Salette J., Lemaire G., 1981. Sur la variation de la teneur en azote des graminées fourragères pendant leur croissance : formulation d'une loi de dilution. C. R. Acad. Sci. Paris, 292, série III, 875-878.

Woodman H. E., Evans R. E., 1935. Nutritive value of lucerne : the leaf stem ratio. J. Agric. Sci., 25, 578-597.

Woodward F. I., Sheehy S. E., 1979. Microclimate, photosynthesis and growth of lucerne. II. Canopy structure and growth. Ann. Bot., 44, 709-719. 